\title{
A Versatile Parameterization for Measured Material Manifolds
}

\author{
Cyril Soler $^{1} \quad$ Kartic Subr $^{2} \quad$ Derek Nowrouzezahrai $^{3}$
}

${ }^{1}$ INRIA - Grenoble University, France $\quad{ }^{2}$ University of Edinburgh, UK $\quad{ }^{3}$ McGill University, Canada
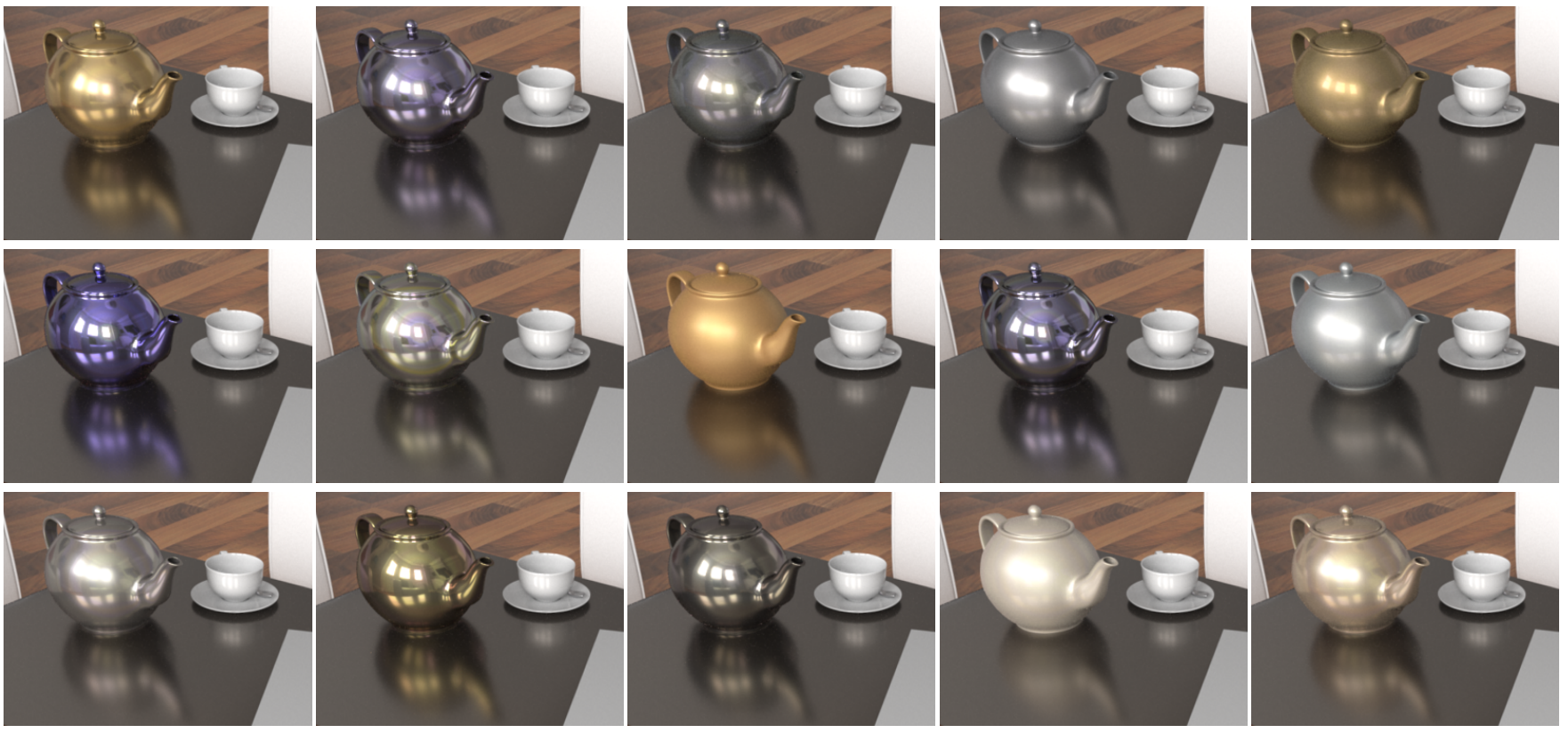

Figure 1: Four of the above images are rendered with measured BRDFs from the MERL dataset [MPBM03]. The remaining 11 are rendered with interpolated BRDFs generated from our parameterization of a non-linear BRDF manifold containing MERL materials. We can explore this manifold to produce realistic BRDFs which interpolate the physical and perceptual properties of the original measured materials.

\begin{abstract}
A popular approach for computing photorealistic images of virtual objects requires applying reflectance profiles measured from real surfaces, introducing several challenges: the memory needed to faithfully capture realistic material reflectance is large, the choice of materials is limited to the set of measurements, and image synthesis using the measured data is costly. Typically, this data is either compressed by projecting it onto a subset of its linear principal components or by applying non-linear methods. The former requires many components to faithfully represent the input reflectance, whereas the latter necessitates costly extrapolation algorithms. We learn an underlying, low-dimensional non-linear reflectance manifold amenable to rapid exploration and rendering of real-world materials. We can express interpolated materials as linear combinations of the measured data, despite them lying on an inherently non-linear manifold. This allows us to efficiently interpolate and extrapolate measured $B R D F s$, and to render directly from the manifold representation. We exploit properties of Gaussian process latent variable models and use our representation for high-performance and offline rendering with interpolated real-world materials.
\end{abstract}

\section{Introduction}

Realistic image synthesis relies on accurate representations of the reflectance of real-world surfaces. A powerful method for obtaining this information is to acquire the bidirectional reflectance distribution function (BRDF) profiles directly from real-world materials.
To do so typically requires capturing and tabulating raw reflectance data from many incident and reflected directions. While the space of BRDFs spanned by these measurements can have an arbitrarily large dimensionality (e.g., four million for the MERL dataset), the subspace of real-world BRDFs is of substantially lower dimensionality [MPBM03]. Directly exploring this subspace, which we call the 
manifold of BRDFs, could lead to exciting applications: real-world BRDF interpolation for material design, BRDF inference, and data completion for partially-observed/sampled BRDFs, to name a few.

Previous approaches apply dimensionality reduction to BRDF data. Linear dimensionality reduction (i.e. PCA variants) require high-dimensional linear spaces (e.g., about 45D for the MERL dataset) to faithfully reproduce measured materials. The same applies to dictionary-based representations $\left[\mathrm{XNY}^{*} 16, \mathrm{HSL}^{*} 17\right]$. When the dimension is low ( 2 to 5 dimensions) neither kernel approximations [MPBM03] nor linear basis function projections [SBN15] are sufficiently accurate for rendering applications. This is unfortunate since linear approximations often yield fast rendering techniques. Standard methods for non-linear dimensionality reduction are not very useful for reasoning about the BRDF manifold: these methods exploit local relations, and so tend to behave poorly at low sampling rates (i.e. few measured BRDFs) and/or noisy measurements - two properties of current BRDF datasets. Charting methods can learn tighter (i.e. 10D) non-linear BRDF manifolds [MPBM03], but they require complex algorithms to extrapolate BRDFs. Euclidean embeddings can provide a latent space that is useful for studying relationships between BRDFs, such as perceptual distances [WAKB09], but they do not permit interpolation and exploration within the space.

One common approach to addressing these shortcomings is to fit non-linear, low-dimensional analytic models to reflectance data and to interpolate directly in the parametric space [NDM05, WMLT07, BSH12,HP17]. Although designing such models is non-trivial, their parameterizations naturally permit interpolation and also lead to low-dimensional manifolds: dimensionality is pre-determined by the model's degrees of freedom. Parametric model fitting can, however, be numerically unstable, especially in the presence of multimodal reflectance profiles common to real-world materials. Another drawback is that these fits provide no smoothness guarantees in the mapping between the parametric space and measured BRDFs. This causes interpolated BRDFs to suffer from abrupt appearance transitions, even when interpolating between similar BRDFs. Finally, realistic rendering with parametric BRDFs still requires costly numerical integration techniques for estimating the rendering (or reflection) equation; typically, these methods propose and rely on importance sampling schemes to accelerate numerical integration, but the integration task remains necessary (see Figure 2).

We use a Gaussian Process Latent Variable Model (GPLVM) to devise a manifold that spans measured reflectance. Our parameterization naturally yields a non-linear manifold that exactly interpolates the input (measured) data. Moreover, as the GPLVM maintains a linear relationship between training and interpolated samples, we can quickly extrapolate BRDFs over the non-linear manifold. We identify and leverage ties in the areas of dimensionality reduction, material appearance and light transport, to:

1. Adapt GPLVM to the problem of optimizing low-dimensional latent spaces that map to the space of measured BRDFs,

2. Use our mapping to traverse points on the non-linear manifold using an efficient linear interpolation of the original BRDFs, and

3. Analyze the stability, physical correctness and plausibility of interpolated/extrapolated BRDFs.

We demonstrate the utility of our BRDF manifold on interactive rendering and approximate global illumination applications.

\begin{tabular}{|r|c|c|c|c|c|c|}
\hline Method & NL & DR & Stab. & Map & Intp. & Eff. \\
\hline PCA & no & poor & good & $H \rightarrow L$ & no & good \\
Dict. & no & none & good & $L \rightarrow H$ & yes & good \\
Kernel PCA & yes & good & good & $H \rightarrow L$ & no & OK \\
MDS & yes & good & good & $H \rightarrow L$ & N/A & OK \\
Isomap & yes & good & good & $H \rightarrow L$ & N/A & poor \\
Parametric & yes & good & poor & $L \rightarrow H$ & no & good \\
Ours & yes & good & yes & $L \rightarrow H$ & yes & good \\
\hline
\end{tabular}

Figure 2: BRDF dimensionality reduction approaches. LEGEND: can capture non-linearities $(N L)$, dimensionality reduction effectiveness $(D R)$, stability, mapping from low-to-high dimensions $(L \rightarrow H)$, interpolation capability (Intp.) and measurement efficiency.

\section{Related Work}

BRDFs maps pairs of directions to real values: $\rho: \omega_{i} \times \omega_{r} \rightarrow \Re$, for each color channel. The BRDF is the ratio of the reflected radiance towards $\omega_{r}$ to the differential radiance from light incident from $\omega_{i}$, at a shading point on a surface. The area of material appearance comprises over four decades of prior art, and we refer readers to comprehensive surveys on the measurement, modelling, analysis and rendering of materials [DRS08, WdBKK15, GGG*16]. Here, we instead focus on works that are most relevant to our problem.

Measuring and modelling acquired BRDFs: Marschner and colleagues [MWL $\left.{ }^{*} 99\right]$ measure real-world materials at many incident and reflected directions, sampled over the 4D domain. Matusik et al.'s dataset includes 100 materials, each sampled with four million direction pairs [MPBM03]. The size of these datasets has motivated more compact representations that trade accuracy for size, such as factorized tabulations [SM02, BSN16], matrix decompositions [KM99], non-negative matrix factorisations [LRR04], inverse shade trees [LBAD*06] and Tucker decompositions [BOK11]. Recent methods capture material properties with impressively few measurements [GVPG15, AAL16, NLW*16, XNY*16], however no large datasets have been created using them.

Analysis: BRDF analysis has been approached from roughly two directions: basis function approximations for individual BRDFs, and larger studies of the entire space of BRDFs. In the former, basis choices include spherical harmonics (SH) [WAT92], spherical wavelets [SS95], clustering-based empirical bases [LKG*03], constrained basis decompositions [LBAD*06] and rotated zonal harmonics [SBN15]. When only partial/sparse observations of a single BRDF are available, Gaussian Process (GP) regression has proven effective for BRDF completion [HLW15] by interpolating a single BRDF in angular space (whereas we interpolate accross BRDFs in a ad-hoc latent space). Radiometric studies of the space of BRDFs apply dimensionality reduction tools directly on the measured data. Linear approaches are unable to identify sufficiently small subspaces [MPBM03] to facilitate practical exploration, whereas many non-linear dimensionality reduction methods (e.g., MDS, ISoMap, LLE) yield compact embeddings without explicitly providing mappings between the measured space and the manifold. Alternatively, one can fit parametric models [NDM05, AP07, BSH12, LKYU12] to the captured data to model variation across measured BRDFs, however fitting can become numerically unstable, especially for materials with multimodal reflectance distributions. 
Perceptual space of BRDFs: Many works aim to understand the perceptual properties of BRDFs, often driven by user studies. This has led to reparameterizations of specific BRDFs with respect to perceptual metrics, as well as identifying semantically meaningful axes of variation for the BRDF manifold (e.g., color and gloss) [PFG00]. Furthermore, correspondences between parametric and perceptual spaces have also been formed [WM01]. Wills et al. [WAKB09] show that linear interpolation in BRDF space does not result in a linear blend of materials in the perceptual space. They obtain an embedding of BRDFs using MDS, rendering images by traversing their embedding. Since MDS cannot map between the two spaces, their method is unable to interpolate materials within the embedding. A recent method identifies an intuitive control space for materials [SGM*16], allowing for impressive perceptually-aware exploration of the acquired material manifold (see Section 6).

Rendering: Although we focus on parameterizing the space of measured BRDFs, we briefly discuss the state of the art in rendering acquired materials since our model also enables rapid rendering. Many methods can directly render acquired materials, from accurate (but slow) physically-based methods to coarser (but faster) approximations. For interactive rendering, the compression and representation of an acquired BRDF is usually tightly coupled to a specialized rendering algorithm. View-light factorization using SVD [KM99] offers a simple rendering algorithm for a specific BRDF, and trilinear tensor factorization extensions [SZC ${ }^{*} 07$ ] improve compression and allow for interactive exploration BRDFs. By projecting BRDFs onto $\mathrm{SH}$, shading can be efficiently reformulated as a scalar product in frequency space for arbitrary 4D BRDFs [SKS02, KSS02]. More recently, an efficient spherical filtering approach using isotropic spherical decompositions [SBN15] has enabled more accurate interactive rendering solutions for arbitrary BRDFs. We demonstrate the versatility of our BRDF manifold by building atop this ISD to enable efficient real-time rendering of interpolated materials. Some of the aforementioned techniques treat visibility under certain constraints, such as static view, geometry, or with costly precomputed representations. Sun et al [SZC*07] additionally treat global illumination using precomputed transfer tensors, and a large body of work on precomputed rendering [Ram09] demonstrate methods that trade speed for accuracy in this domain. Xu et al [XCM*14] render 1-bounce interreflections, however it is unclear how their spherical-Gaussian representation can be used to realistically render measured BRDFs.

\subsection{An Overview of Gaussian Processes}

A Gaussian process (GP) is a collection of random variables, any finite number of which stem from a joint Gaussian distribution. If the random values represent evaluations of a function $f: X \rightarrow \Re$, their associated GP implicitly models distributions over the space of functions. Here, we overview how to use GPs to perform regression and optimize low-dimensional latent variables. We limit our discussion to an extent necessary for understanding our problem, and we refer interested readers to more comprehensive surveys [RW06].

Regression: Our goal is to predict the value $z_{*}$ at arbitrary locations $\mathbf{x}_{*} \in X$, given pairs of observed values $\left(\mathbf{x}_{i}, z_{i}\right)$ at training locations $\mathbf{x}_{i} \in X$, where $i=0,1, . . N-1$. By definition, if we denote the vector of values $\mathbf{z}=\left[z_{0}, z_{1}, \ldots, z_{N-1}\right]^{\top}$ of the GP, then it follows that $\mathbf{z} \sim \mathcal{N}\left(\mu_{z}, \mathbf{K}\right)$, where $\mu_{z}$ and $\mathbf{K}$ are the mean and covari-

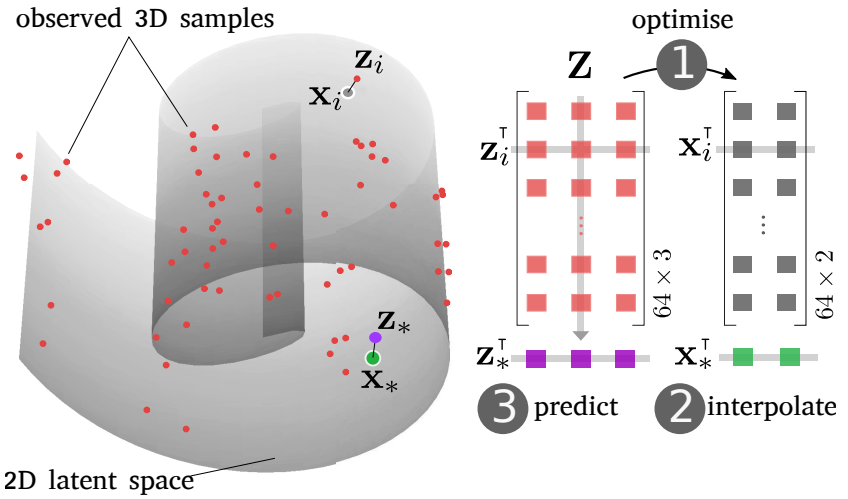

Figure 3: Illustration of a $2 D$ latent space (grey surface) of $643 D$ measurements (red dots); so $N=64, d=3$ and $q=2$. We also show the latent variables associated with the measurements (grey dots) and interpolated latent variable (green dot) and the interpolated measurement (purple dot).

ance of the Gaussian distribution. The covariance matrix elements $\mathbf{K}_{i j}=c\left(\mathbf{x}_{i}, \mathbf{x}_{j}\right)$, where $c: X \times X \rightarrow \Re$ is a user-defined covariance function. Here, $c$ can be interpreted as a kernel, and it is key to modeling the non-linearity of the underlying function. The consistency (or marginalization) property of GPs states that "slicing" a GP along any 1D subspace of coordinates results in a 1D Gaussian distribution. So, $z_{*} \sim \mathcal{N}\left(\mu_{z *}, \sigma_{z *}^{2}\right)$, with [RW06]:

$$
\begin{aligned}
\mu_{z_{*}} & =\mathbf{k}_{*}^{\top} \mathbf{K}^{-1} \mathbf{z}, \\
\sigma_{z^{*}}^{2} & =c\left(\mathbf{x}_{*}, \mathbf{x}_{*}\right)-\mathbf{k}_{*}^{\top} \mathbf{K}^{-1} \mathbf{k}_{*} \text { and } \\
\mathbf{k}_{*} & =\left[c\left(\mathbf{x}_{0}, \mathbf{x}_{*}\right), c\left(\mathbf{x}_{1}, \mathbf{x}_{*}\right), \ldots, c\left(\mathbf{x}_{N-1}, \mathbf{x}_{*}\right)\right]^{\top} .
\end{aligned}
$$

Interpolation is formulated as a prediction of output values $\mu_{z *}$ that requires: evaluating $\mathbf{k}_{*}$, the input covariance function between each training (observed) and test location, solving for $\mathbf{K}^{-1} \mathbf{z}$ (a linear system of dimension $N$ ), and computing an inner product of two vectors. This method models non-linearities by virtue of the nonlinearity of the covariance function. We extend single-output variable case $z$ (resp. $z_{i}$ ) to a $d$-dimensional output, where the function being learned is $f: X \rightarrow \mathfrak{R}^{d}$, by simply replacing the observation vector $\mathbf{z}$ with an observation matrix $\mathbf{Z}$ in Eq. 1, each column of which is independently extrapolated (Step 3 in Figure 3.)

Latent Variable Model (LVM): In our situation, $\mathbf{z}_{i} \in \mathfrak{R}^{d}$ are observed but the corresponding $\mathbf{x}_{i}$ are unknown: e.g., each $\mathbf{z}_{i}$ could have $d=4$ million measurements of a single acquired BRDF. Although $\mathbf{x}_{i} \in \mathfrak{R}^{q}$ may be chosen arbitrarily and associated with corresponding $\mathbf{z}_{i}$, the interpolation at some $\mathbf{x}_{*} \in \mathfrak{R}^{q}$ is not expected to be useful since $\mathbf{K}$ (the covariance function evaluated at all-pairs of the chosen $\mathbf{x}_{i}$ ) is implicitly dependent on the arbitrarily chosen $\mathbf{x}_{i}$. Although the "kernel trick" may be used in conjunction with PCA for learning non-linear mappings from observed to latent variables, they are not easily invertible. For our application of exploring the manifold of BRDFs, we seek a dual of kernel PCA, which learns the mapping from the latent space to the space of measurements (rather than the other way around). GPLVM [Law05] achieves this by optimizing the likelihood of the latent variables $\mathbf{x}_{i}$ given the measured data, under a GP prior on the mapping (a given covariance 


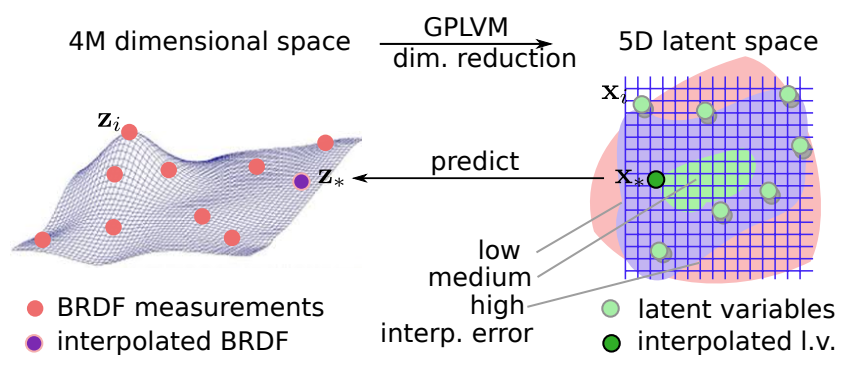

Figure 4: We use GPLVM to identify a low-dimensional non-linear manifold of measured BRDFs, parameterized by latent variables. We interpolate the latent variables and map interpolated vectors to the data space, obtaining an interpolated $B R D F \mathbf{z}_{*}$ that is a linear combination of observations $\left\{\mathbf{z}_{i}\right\}$.

function). The non-linearity in the mapping is governed by the covariance function. A key property that we exploit in our work is that the interpolations remain linear with respect to the observations.

Linearity of interpolation using GPLVM: Let $\mathbf{Z}_{N \times d}$ be the matrix of $N$ observations stacked so that the $i^{\text {th }}$ row is $\mathbf{z}_{i}^{\top}$ and the $j^{\text {th }}$ column is a vector composed of the $j^{\text {th }}$ dimensional components of all $N$ observations. The output of GPLVM is $N$ optimised $q$ dimensional latent variables $\mathbf{x}_{i}$. Then, the problem of traversing the manifold (latent space) is identical to regression. Given some traversal location $\mathbf{x}_{*}$, the goal is to predict the corresponding extrapolated observation $\mathbf{z}_{*}$ (akin to Eq. 1)

$$
\mathbf{z}_{*}^{\top}=\mathbf{b}_{x *}^{\top} \mathbf{Z}
$$

where $\mathbf{b}_{x *}^{\top}=\mathbf{k}_{*}^{\top} \mathbf{K}^{-1}$. Although $\mathbf{b}_{x *}$ is non-linear with respect to the latent variables $\mathbf{x}_{i}$, the extrapolated data remains linear with respect to the observed data $\mathbf{Z}$

Properties: To summarize, we choose the latent space generated by GPLVM because it offers the following key properties: non-linear mapping from an optimized, low-dimensional latent space to highdimensional observations; linearity with respect to observations; guaranteed interpolation of observed data regardless of the latent variables $\mathbf{x}_{i}$ (if $\mathbf{x}_{*}=\mathbf{x}_{i}$, then Eq. 4 yields $\mathbf{z}_{*}=\mathbf{z}_{i}$ ); continuity in the interpolated observations for continuous covariance functions; flexibility in the dimensionality of the latent variable space.

\section{Parameterizing the BRDF Manifold}

We learn the manifold of acquired BRDFs using GPLVM (reviewed in sec. 2.1). The MERL dataset [MPBM03] contains $N=100$ materials, each with $d=4 M$ (four million) measurements. Each measurement records a scalar measurement of the reflectance for a specific pair of incident and reflected directions. Thus, the size of our observation matrix $\mathbf{Z}$ is $100 \times 4 M$. We first subtract the mean $B R D F$ from the entire training set, so that extrapolated values far from the input data converge to the average of all input BRDFs (instead of to 0). We calculate optimized latent variables, obtaining a manifold where the spacing between latent variables is in accordance to the variation of the data. The output of this step is a matrix $\mathbf{X}$ of size $100 \times q$ whose rows are the latent variables $\mathbf{x}_{i}^{\top}$. Best results are obtained with $q=5$ for the full MERL database although $q=2$ used in our video, for easy illustration, yields excellent results. Smaller sets of similar materials are very well approximated with $q=2$.

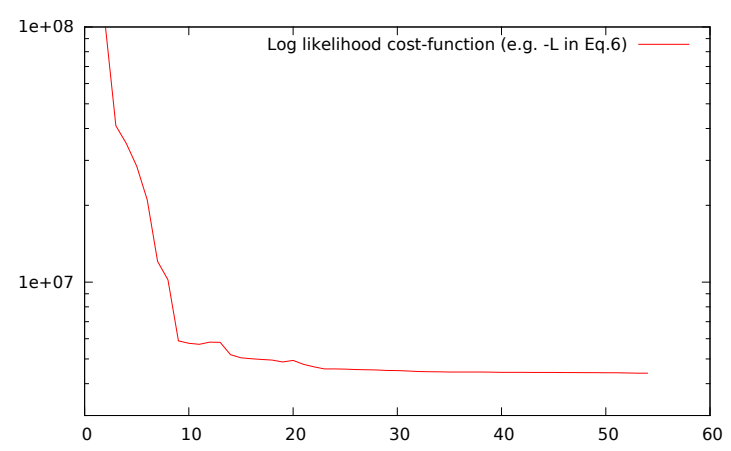

Figure 5: Convergence of the log-likelyhood when fitting the full MERL database using a latent space of dimension 2

Choice of covariance function: We use a shifted squaredexponential function (widely used in the GP literature),

$$
c\left(\mathbf{x}, \mathbf{x}^{\prime}\right)=\mu \delta\left(\mathbf{x}, \mathbf{x}^{\prime}\right)+e^{-\left\|\mathbf{x}-\mathbf{x}^{\prime}\right\|^{2} / 2 \ell^{2}},
$$

where $\ell$ and $\mu$ are hyperparameters that correspond to the characteristic length scale and noise-filtering parameter respectively. Since the expected number of level-zero upcrossings for a 1D stationnary GP with this covariance function is $(2 \pi \ell)^{-1}$, a high value for $\ell$ leads to a smoother function (see, e.g., [RW06, chap. 4]). A small value for $\mu\left(10^{-4}\right)$ significantly improves numerical stability when inverting $\mathbf{K}$ at the cost of introducing a negligible discontinuity in the interpolant. We choose this covariance function because of its smoothness and local support, which translates into both smooth transitions across observed BRDFs (see discussion in Sec. 4.1) and limited, controllable local influence of neighboring BRDFs.

Optimization: We obtain optimised latent variables $\left\{\mathbf{x}_{i}\right\}$, by maximising the log-likelihood of the GP for a fixed choice of $\ell$ and $\mu$ :

$$
L=-\frac{d}{2} \log |\mathbf{K}|-\frac{1}{2} \operatorname{tr}\left(\mathbf{K}^{-1} \mathbf{Z} \mathbf{Z}^{T}\right)
$$

We perform this optimisation using direct local search [HJ61] which offers an efficient calculation scheme in our case since this only requires evaluating the cost function $L$ once per variable $\mathbf{x}_{i}$. We maintain both the inverse and the determinant while changing each row and column of $\mathbf{K}$ using the Sherman-Morisson [PTVF07] and matrix determinant [Har97] formulas twice each. Figure 5 shows the evolution of the log-likelihood, over successive iterations, when fitting the full MERL database with $q=2$. The corresponding map is displayed in Figure 10. We do not perform complicated optimisation methods such as scaled conjugate gradients (gradients of the log-likelihood may be calculated using the chain rule), because the computational complexity of gradient-based methods scales cubically with $N$. We initialise the latent variables using truncated linear PCA. See sec. 6 for a discussion of these choices.

Note that, in this setup, $\ell$ can be chosen arbitrarily, since scaling $\ell$ and latent points $\mathbf{x}_{i}$ simultaneously will not affect the covariance nor the $\log$-likelihood. We therefore set $\ell=1$. The second hyperparameter $\mu$ is only needed to maintain a well-conditioned $\mathbf{K}$, and we set it to $\mu=10^{-4}$ in all our experiments.

Interpolating materials: Given the latent variables $\mathbf{x}_{i}$ from the 
previous step and a new location $\mathbf{x}_{*}$, we calculate the interpolated BRDF (observed) using eq. 4 . The choice of $\mathbf{x}_{*}$ depends on the application for which the BRDF manifold needs to be traversed. For interactive exploration of the space of acquired BRDFs, we either limit ourselves to 2D latent spaces or display 2D slices of 5D latent spaces along with their corresponding projections of $\mathbf{x}_{i}$. The user then manually selects and drags a point within this subspace as $\mathbf{x}_{*}$ with real-time interpolation of the BRDFs. Further, we also tested interpolations between two chosen BRDFs along the 1D trajectory between them in the latent space.

\section{Evaluation}

\subsection{Behavior of the interpolant}

We wish to bound variations in the interpolated data $\mathbf{z}_{*}$ when our mapping is applied to $\mathbf{x}_{*}$ in latent space. Having already established that $\mathbf{z}_{*}$ interpolates measured data $\mathbf{z}_{i}$ when $\mathbf{x}_{*}=\mathbf{x}_{i}$, we study the interpolant at other regions in the latent space. Equation 4 suggests that the quality of the interpolant depends on the covariance function and the inverted matrix $\mathbf{K}^{-1}$. More precisely, the behavior of the interpolant is dictated by two factors: the chosen hyperparameters ( $\ell$ in our case) as well as the particular choice of $\left\{\mathbf{x}_{i}\right\}$.

Using a first-order approximation of Gaussian covariance functions, we can prove (see supplemental material) that every choice of $\mathbf{x}_{*}$ in the latent space leads to a mapping $\mathbf{z}_{*}$ that can be connected to at least one of the training data points using:

$$
\left\|\mathbf{z}_{*}-\mathbf{z}_{j}\right\| \leq \frac{e^{-1 / 2} \sqrt{2 N}}{\ell} \kappa(\mathbf{K})\|\mathbf{Z}\|\left\|\mathbf{x}_{*}-\mathbf{x}_{j}\right\| .
$$

Here, $\mathbf{z}_{j}$ (where $j=\beta\left(\mathbf{x}_{*}\right)$ depends on $\mathbf{x}_{*}$ ) is the latent variable associated with one of the input measurements (most of the time, corresponding to the closest point in latent space), $\|$.$\| is the L_{2}$ norm on vectors (Frobenius norm on matrices) and $\kappa(\mathbf{K})$ the condition number of $\mathbf{K}$. Eq. 7 shows that it is desirable to optimize the ratio between the density of latent variables and the hyperparameter $\ell$. We do this by keeping $\ell$ fixed and optimizing the log-likelihood of the latent variables given the measured data under the GP prior. The motivation for this choice is that RHS of Equation 6 reduces the condition number, since the eigenvalues of $\mathbf{K}$ are less than 1 .

Our bound can be verified to be zero when $\mathbf{x}_{*}$ is one of $\mathbf{x}_{i}$ since $\beta\left(\mathbf{x}_{i}\right)=i$. Another way to interpret the role of $j=\beta(\mathbf{x} *)$ in Equation 7 is that the interpolant stays close to the value at training data points proportional to $\|\mathbf{Z}\| \kappa(\mathbf{K}) / \ell$. From equation 7, it follows that choosing $\ell$ to be very small or very large causes the maximum deviation to worsen. The bound can be tightened for the special case that $\ell$ approaches zero. In this case, which is of purely academic interest, the interpolated BRDF is the average of all measurements everywhere in the domain except very close to the $\mathbf{x}_{i}$ (See Fig.6). We derive (in supplemental material) a tighter bound of the same nature by considering higher-order derivatives of the covariance function.

\subsection{Physical correctness}

Since the interpolated data is linear in the measurement matrix $\mathbf{Z}$ (eq. 4), any properties defined using linear operators of the measured data are preserved after mapping from $\mathbf{x}_{*}$. Therefore, the interpolated

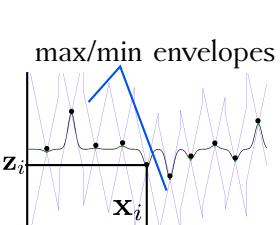

(a) small $\ell$

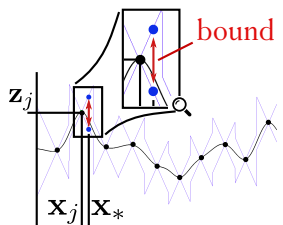

(b) medium $\ell$

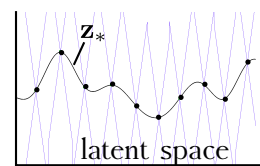

(c) large $\ell$
Figure 6: Illustration of the bound on the behavior of the interpolant derived using a first order approximation.

BRDF (1) obeys Helmholtz reciprocity; (2) implicitly interpolates albedo and (3) applies to reflectivity measurements along a fixed direction of incidence.

The Gaussian process does not provide a theoretical guarrantee that the albedo of interpolated BRDFs will be limited to $[0,1]$. However, the optimization of the log-likelyhood inherently limits the oscillations of the interpolant in between observed data because it tends to keep $\kappa(\mathbf{K})$ as small as possible, tightenning the bound in Eq. 7. To illustrate this, we visualize the albedo of the interpolated BRDFs in the extreme case where we learned a 2D manifold of the entire MERL database. Figure 7 shows a portion of the manifold (for clarity) along with iso-lines of albedo. We define albedo as the ratio between the total amount of radiance reflected by a material under a uniform incident distribution and the irradiance of that distribution:

$$
a(\mathbf{x})=\frac{1}{\pi} \int_{\Omega} \int_{\Omega} f_{\mathbf{x}}\left(\omega_{i}, \omega_{o}\right)\left(\omega_{i} \cdot \mathbf{n}\right) \mathrm{d} \omega_{i} \mathrm{~d} \omega_{o},
$$

where $f_{\mathbf{x}}$ is the interpolated BRDF obtained by mapping $\mathbf{x}$ into the observation space, $\mathbf{n}$ is the vertical axis, and $\Omega$ the upper hemisphere of incident directions. Every pixel in figure 7 corresponds to the albedo of the interpolated BRDF at the corresponding 2D point of the BRDF manifold. The albedo can be observed to remain stable in the entire domain. For latent points $\mathbf{x}_{*}$ that are far away from the training data, the interpolated BRDF $\mathbf{z}_{*}$ approaches the average of all training data thus preventing unexpected oscillations.

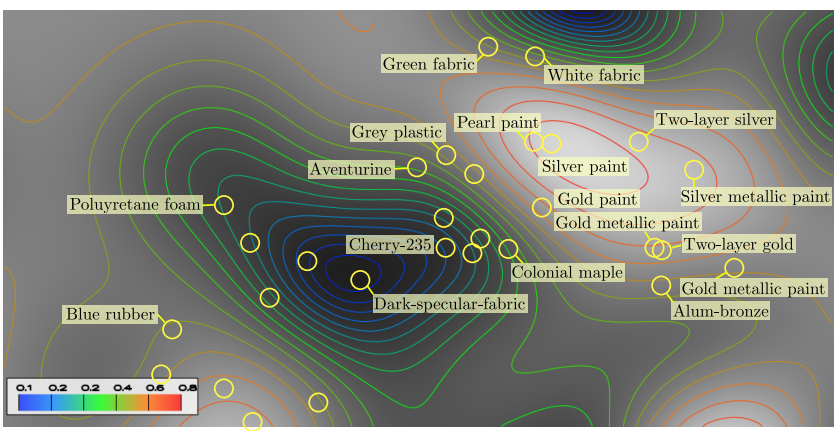

Figure 7: Zoomed region of a 2D manifold of MERL BRDFs juxtaposed on a map of the of the interpolated BRDFs and its iso-curves. The albedo of our interpolated model is observed to be limited to $[0,1]$ and it smoothly interpolates the albedo of the input materials.

Unlike kernel PCA (or any linear decomposition over a set of basis functions), our method linearly interpolates training data. Regardless of the dimensionality of the latent space, our method will always faithfully reproduce the measured BRDF data. Thus, even 


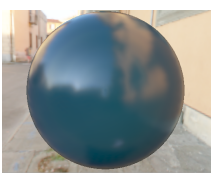

PCA, dim. 20

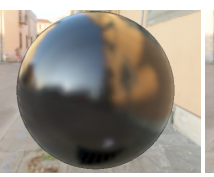

PCA, dim. 40

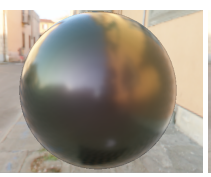

Ours, dim. 2

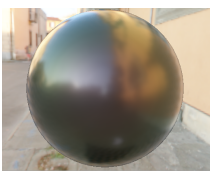

MERL data

Figure 8: Comparing our method to the "kernel trick" when reproducing input BRDF data for a material in the MERL database (color-changing-paint3). Our method always perfectly reproduces the training materials, regardless of the dimension of the parameter space; this is not the case for kernel approximations (here, using 20-and 40-dimensional PCA approximations).

with a manifold of dimension 2, we can perfectly interpolate the whole MERL database. A PCA approximation of the same data with subspaces up to dimension 40 are unable to accurately reproduce measured (input) BRDFs; a minimum of 50 to 60 eigenfunctions [MPBM03] are required for most materials in this database.

\subsection{Plausibility of interpolated BRDFs}

Our choice of the manifold is not perceptually motivated. Although our main contribution is to present objective and empirical benefits of the parameterization, we also assessed our interpolated BRDFs using simple qualitative as well as quantitative experiments. The accompanying video shows several objects rendered in real-time with interpolated BRDFs corresponding to various points selected interactively in our manifold. These experiments are consistent with the behavior that we observed, that the resulting materials remain plausible everywhere in the latent space. Figure 1 shows images rendered using 11 materials chosen from randomly sampled points in the latent space and 4 images corresponding to actual BRDFs that were present in the MERL database. It is not obvious that the 4 measured materials are: row 1, column 4 (two-layer silver); row 2, column 1 (chrome-steel); row 2, column 3 (gold-paint); and row 3, column 2 (brass). While a thorough perceptual evaluation would require user studies, which are beyond the scope of our work, this initial experiment suggests that our interpolated materials are plausible.

To quantitatively study the behaviour of interpolated BRDFs, we use interpolating latent space paths between arbitrary pairs of BRDFs, dividing them into 20 equal step intervals. At each of these keypoints, we compute the perceptual distance [PR12] of the interpolated BRDF from the source and destination BRDFs. Figure 9 shows three examples: (a) blue-metallic-paint to dark-blue-paint; (b) teflon to silver-paint; and (c) black-oxidized-steel to color-changing-paint3. The blue and red curves are distances of the interpolated BRDFs to the source (BRDF A) and destination (BRDF B); ideal (i.e., perceptually uniform) behavior is shown using dashed lines. Figure $9(\mathrm{c})$ is affected by the spectral behavior of color-changing-paint 3.

\subsection{Computation time}

The computational complexity of a single latent variable fitting step is $O\left(N^{2}\right)$. For the full MERL database, the total fitting time on a single core of an Intel i7 processor is 122 seconds. For interpolation, we precompute and store $\mathbf{K}^{-1} \mathbf{Z}$ for the training data. Therefore,

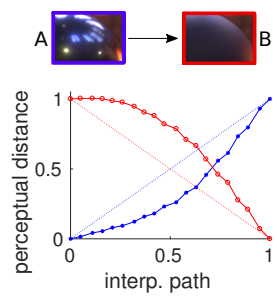

(a)

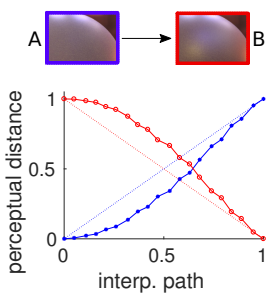

(b)

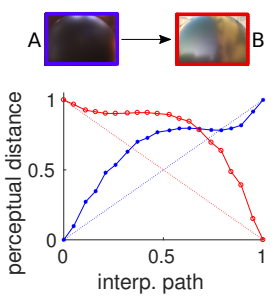

(c)
Figure 9: Perceptual distances [PR12] (Y-axis) between interpolated materials (data points) at regular distances in our latent space (dimension 5), between BRDF A (blue) and BRDF B (red). The $X$ and $Y$ - axes are normalized by the distance between $A$ and $B$ and the perceptual distance between $B R D F A$ and $B R D F B$, respectively.

computing an interpolated BRDF amounts to calculating the correlation vector $\mathbf{k}_{*}$ ( $N$ covariance function evaluations) followed by a matrix-vector multiplication $\mathbf{K}^{-1} \mathbf{Z}$. All together, this takes less than 1 second on a single CPU core. For rendering, we instead interpolate much smaller data (e.g., zonal harmonic BRDF coefficients; see Section 5.1), for which the computation time is negligible.

\section{Use cases}

We demonstrate the utility of our BRDF manifold parameterization in different scenarios, seamlessly integrating our extrapolation method into existing rendering techniques.

\subsection{Interactive BRDF manifold exploration}

Estimating the reflectance integral, that describes the radiance arriving from a point $\mathbf{r}$ along a direction $\omega_{o}$ towards the centre of projection through pixel $\mathbf{p}$, is fundamental to realistic image synthesis applications:

$$
I\left(\mathbf{p}, \omega_{o}\right)=\int_{\mathcal{S}^{2}} L(\mathbf{r}, \omega) \rho\left(\mathbf{r}, \omega_{o}, \omega\right) v(\mathbf{r}, \omega) \max (0, \omega \cdot \mathbf{n}) \mathrm{d} \omega,
$$

where $L(\mathbf{r}, \omega)$ is the incident radiance at $\mathbf{r}$ along $\omega, \rho$ is the BRDF at $\mathbf{r}, v(\mathbf{r}, \omega)$ is the visibility of the source of $L$ at $\mathbf{r}$ along direction $\omega$ and $\mathbf{n}$ is the normal at $\mathbf{r}$.

Fixed view: Given a fixed viewing direction $\omega_{o}$, we can render a pixel $\mathbf{p}$ with a BRDF from the manifold as

$$
I_{*}(\mathbf{p})=\sum_{i=1}^{N} \mathbf{b}_{x *}^{i} I_{i}(\mathbf{p})
$$

due to the linearity of Equation 8 w.r.t. $\rho$ and since any material generated by our manifold parameterization is a linear combination of the $N$ measured BRDFs (eq. 4): $\rho_{*}=\sum_{i=1}^{N} \mathbf{b}_{x *}^{i} \rho_{i}$, where $I_{i}$ is the image rendered with training material $\rho_{i}$. For some applications, such as material design, pre-rendered images (one image for BRDF in the training set) may be used to explore the interpolated appearances on the BRDF manifold without recalculating the reflectance integral for a fixed view. Images using interpolated materials can be obtained by linear interpolation of the pre-rendered images using $\mathbf{b}_{x *}$ as coefficients.

Dynamic view/geometry/lighting: Using the above argument, any algorithm that expresses the BRDF (and hence the image itself) by projection onto a linear basis can be adapted to use our interpolated 
BRDF with minimal implementational changes. We demonstrate this using the example of a recent algorithm [SBN15] which expresses the BRDF as a sum of rotated zonal harmonics (RZH) - special spherical harmonics $(\mathrm{SH})$ that are invariant to rotations through a particular fixed axis. This paper exploits the property that a statically chosen set of $\left(l_{\max }+1\right)^{2} \mathrm{ZH}$ along $2 l_{\max }+1$ fixed axes $\mathbf{a}_{m}$, where $l_{\max }$ is the degree, together form a basis that exactly spans the space of SH. Using this, the authors compute the shading equation in real time for large values of $l_{\max }$. For directional (distant) lighting, where $L(\cdot, \omega)=E(\omega)$ (temporarily ignoring the visibility term for simplicity), they derived the reflectance equation

$$
I(\mathbf{p}, \omega)=\sum_{l=0}^{l_{\max }} \sum_{m=-l}^{l}\left(E \otimes \mathcal{Y}_{l}^{0}\right)\left(\mathbf{R}_{n}^{-1} \mathbf{a}_{m}\right) \lambda_{l}^{m}\left(\mathbf{R}_{n} \omega_{o}\right) .
$$

Here, $\mathbf{R}_{n}$ is a rotation that maps directions expressed in a global coordinate system to those in a local coordinate system turning $\mathbf{n}$ into $(0,0,1), E \otimes \gamma_{l}^{0}$ denotes spherical convolution of the illumination with a zonal harmonic $\mathcal{Y}_{l}^{0}$, and $\lambda_{l}^{m}$ are RZH coefficients of the BRDF. Since $\lambda_{l}^{m}$ linearly depends on the BRDF, there exists a constant matrix $\mathbf{P}_{\mathbf{a}}$ (that depends only on directions $\left\{\mathbf{a}_{m}\right\}$ ), so that the vector $\Lambda_{i}^{\top}$ of the $\left(l_{\max }+1\right)^{2}$ zonal harmonic coefficients associated with reflectance $\rho_{i}$ is $\Lambda_{i}^{\top}=\mathbf{z}_{i}^{\top} \mathbf{P}_{\mathbf{a}}$. The interpolated ZH coefficients corresponding to $\rho_{*}$ are consequently

$$
\Lambda_{*}^{\top}=\mathbf{z}_{*}^{\top} \mathbf{P}_{\mathbf{a}}=\mathbf{b}_{x *}^{\top} \mathbf{Z} \mathbf{P}_{\mathbf{a}}=\mathbf{b}_{x *}^{\top} \Lambda
$$

where the matrix $\Lambda$ is formed by stacking the $\Lambda_{i}^{\top}$ as its rows. So $\Lambda_{*}$ can be computed without the need for explicitly involving $\mathbf{P}_{\mathbf{a}} \cdot \Lambda$, which is used as $\mathbf{Z}$ in Equation 4, is $N \times\left(l_{\max }+1\right)^{2}$. Given that $\Lambda_{*}$ is the set of $\mathrm{ZH}$ coefficients for the interpolated material, we simply supply the shader of Soler et al. [SBN15] with the interpolated material's $\Lambda_{*}$ for real-time rendering. Due to this simplicity, our interpolation can be used with either variant of their real-time shader: static geometry with the visibility term or dynamic geometry but without visibility. This technique was used to display the dragons in Figure 10 at $25 \mathrm{fps}$ (See also our accompanying video for an interactive example) using a 2D parameterization of the manifold from the full MERL data set [MPBM03].

\subsection{Real-time material editing with global illumination}

Assuming the shading point $\mathbf{r}$ resides on an object whose material $\rho$ we wish to modify, we separate the paths of light arriving at $\mathbf{r}$ into two classes $L(\mathbf{r}, \omega)=L_{1-}(\mathbf{r}, \omega)+L_{2+}(\mathbf{r}, \omega)$ based on whether the paths bounce at most once $\left(L_{1-}\right)$ on $\rho$ or twice or more $\left(L_{2+}\right)$ as depicted in figure 11. Since $L_{1}$ - contains paths with at most one interaction involving $\rho$, its contribution to $L\left(\mathbf{r}, \omega_{o}\right)$ is affine in $\rho$. Substituting this in eq. 8 results in a separation of the image $I$, where the material to be modified is $\rho$, into $I=I^{1-}+I^{2+}$ where $I^{1-}$ is an image that is entirely affine in $\rho$ and $I^{2+}$ contains the remainder of the energy. Due to this linearity, by construction,

$$
I_{i}^{1-}(\mathbf{p}, \omega)=T \mathbf{z}_{i}
$$

where $T$ corresponds to a non-conventional form of the transport matrix. Rather than expressing the radiance at the image plane through linear transport from the light source, Equation 12 represents the image as a linear combination of the measured $4 \mathrm{D}$ reflectance data for light bouncing at most once on $\rho_{i}$. $T$ includes information about the geometry and lighting in the scene. Note that this is different from direct reflection because $T$ includes multibounce paths to the exception of paths that contain more than 1 reflection off the surface with the changed material. Due to linearity, the image $I_{*}^{1-}$ where $\rho$ is replaced with $\rho_{*}$ is obtained by interpolating pre-rendered images:

$$
I_{*}^{1-}(\mathbf{p}, \omega)=\sum_{i=1}^{N} \mathbf{b}_{x *}^{i} I_{i}^{1-}(\mathbf{p}, \omega) .
$$

In practice we expect $L_{2+} \ll L_{1-}$, since the measure of multiplebounce paths for which more than one of the bounces on $\rho$ is expected to be small. We observe that applying this interpolation to calculate $I_{*}$ directly rather than $I_{*}^{0}$ produces very plausible results. A measurement of the approximation error is shown in Figure 13.

Further, Figure 12 visualizes screenshots from a live session where the user interpolates in the manifold between two BRDFs from the training set. The images using interpolated materials (shown alongside) are rendered in real time by blending images that were pre-rendered using training data (measured materials) with global illumination. Interpolation coefficients are computed in real time and applied to the precomputed images, resulting in blended images that are accurate approximations of images obtained by solving for global illumination with the interpolated material.

\subsection{Importance sampling}

Although the above use cases do not require numerical integration, the ability to draw samples distributed according to 2D slices of BRDF may be useful for general rendering approaches that wish to use our representation of the BRDF manifold. There are multiple ways of importance sampling our interpolated BRDFs. The straightforward way would be to exploit linearity and interpolate precomputed cumulative distribution functions (CDFs) associated with each of the materials. The CDF of the interpolated BRDF slice is easily computed on-the-fly. This method, although simple to implement, would incur an extra cost of numerical inversion of the $\mathrm{CDF}$ while sampling. Some renderers generate importance samples by first fitting parametric models (with prescribed importance sampling algorithms) to the acquired BRDFs. In that case, the parameters for each $\mathbf{z}_{i}$ could be set as the latent variables $\mathbf{x}_{i}$. Instead of generating optimized latent variables we would then optimize the hyper-parameters. The resulting $\mathbf{x}_{*}$ would correspond to the parameters for the interpolated BRDF and importance sampling could be performed as prescribed by the chosen parametric model.

\section{Discussion}

Comparison to BRDF interpolation methods: Existing point-topoint BRDF interpolation methods [BvdPPH11] are excellent at interpolating between pairs of materials. However they do not provide a full parameterization of the BRDF manifold, which makes it impossible to explore the dataset outside the "optimal path" computed by the interpolation method.

Other work using GPs: GPs are popular tools that have been widely used. As explained in sec. 2 GPs have been explored for regression to complete missing BRDF data [HLW15] for a single BRDF. Georgoulis used GPs to overcome the problem of illposedness while performing BRDF inference [GVPG15], by working in the (much smaller) latent space. In this paper, we exploit the 

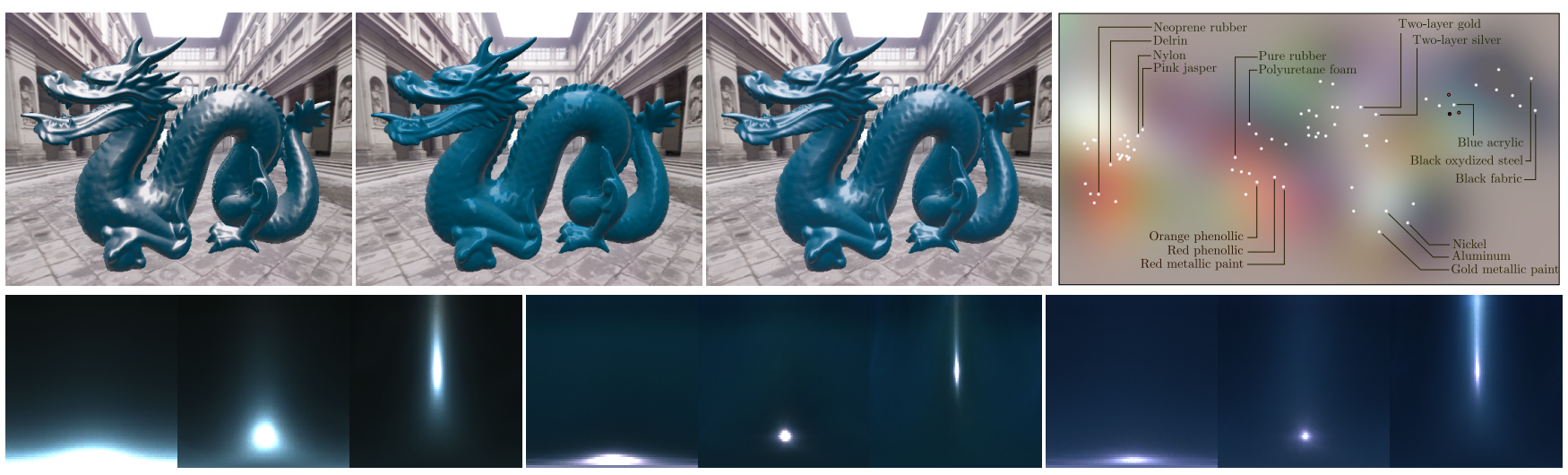

Figure 10: Real-time exploration of our BRDF manifold. 3 materials (circle points) are picked up in the vicinity of bl ue-acry 1 ic, by clicking in the 2D latent space (top right), showing consistent local variations of both color and shininess. Here latent variables were optimised using all 100 materials of the MERL database. The dragons are screenshots from our real-time rendering (25 fps) prototype, which shades interpolated materials along with all combinations of dynamic geometry, view points and lighting (see sec. 5.1) using zonal harmonics up to $L=40$ [SBN15]. The slices of the interpolated BRDFs are also visualised. A live demonstration is presented in our video.

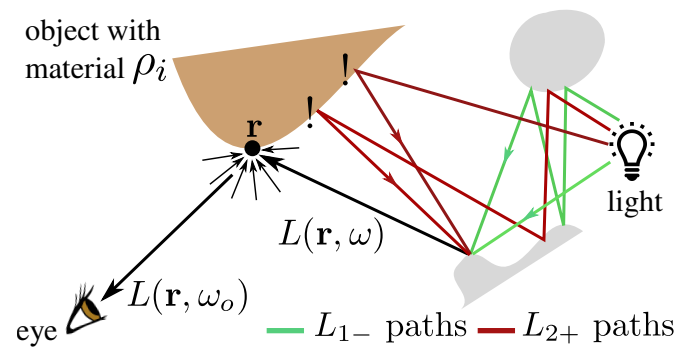

Figure 11: An illustration of multi-bounce paths contributing to the reflectance integral at a point $\mathbf{r}$ on a material $\rho_{i}$ which we wish to modify. We seggregate contributions to $L(\mathbf{r}, \omega)$, the radiance along one incident direction $\omega$ at $\mathbf{r}$, into $L_{1-}$ (green) and $L_{2+}$ (red) based on whether the paths contain at most one point on object with $\rho_{i}$ before arrival, or more: $L(\mathbf{r}, \omega)=L_{1-}(\mathbf{r}, \omega)+L_{2+}(\mathbf{r}, \omega)$.

linearity of the interpolated variables with respect to the observed data for computer graphics applications.

Editing multiple materials simultaneously: Our discussion through the paper has been focused on modifying one of the BRDFs in the scene. This trivially generalises to real-time rendering of multiple materials using $\mathrm{ZH}$, for materials either on the same manifold or on different manifolds. For interpolation of pre-rendered GI images, editing $p$ materials requires a multi-linear interpolation of dimension $p$, applying Equation 13 to compute intermediate points.

Anisotropic materials: Assuming that the measurement spaces are consistent over the different measured data, there is no fundamental difference in using anisotropic BRDFs. A larger set of measurements would simply increase the size of vectors in Eq.4 and our approach would consume larger computational resources. Both use-cases, the real-time applications as well as GI interpolations, naturally extend to anisotropic BRDFs. The consequences of the larger memory footprint of measurements is not a problem for the former since the training data is never stored in memory; only the ZH coefficients of the interpolated BRDF are stored on the GPU.
Colored materials: There is no trivial way to separate spectral behavior of measured BRDFs. Materials such as color-changing-paint 3, for instance, do not have a meaningful average color. We concatenate all three channels into the observation (data) matrix $\mathbf{Z}$, thus tripling the dimensionality of measurements. We experimented with both RGB and CIE Lab without significant differences and therefore used concatenated RGB measurements for all experiments in this paper.

Use in material design: Our method inherently interpolates BRDFs from the training database while retaining their physical properties and perceptual aspects, producing very realistic results that we believe suit material design. If the manifold is learned by omitting certain measured materials, it still retains its effectiveness for exploration if the omitted materials can be expressed as linear combinations of the training materials. Of course, this is no longer the case if the omitted materials are independent of the other measurements. The extrapolated BRDFs at points on the manifold that are far from all training data points represent the "average" BRDF, corresponding to the mean of the measurements from training data. Since our goal is to identify the non-linear manifold of measured data, we selected key-points for interpolated BRDFs arbitrarily without addressing perceptual attributes. Recent work on exploring the intuitive space of materials [SGM* 16 ] uses data from user-studies to learn non-linear mappings from the top 5 principal components to perceptually-meaningful attributes. They demonstrate impressive applications such as artistic exploration of the space of plausible materials. However, since their mapping to the perceptual attributes is non-linear and their interpolated BRDFs are non-linear in the measurements, their method does not lend itself to efficient rendering. We hope that our manifold will inspire future research towards perceptually uniform traversal of measured BRDF manifolds.

Choice of dimensionality: While the training data is always reproduced exactly whatever the dimension of the latent space, the consistentency of interpolation between materials depends on whether the path in latent space has other materials "nearby". While choosing a low-dimensional space (e.g. 2D) is advantageous for interactive editing, it also increases the probability of finding training points 

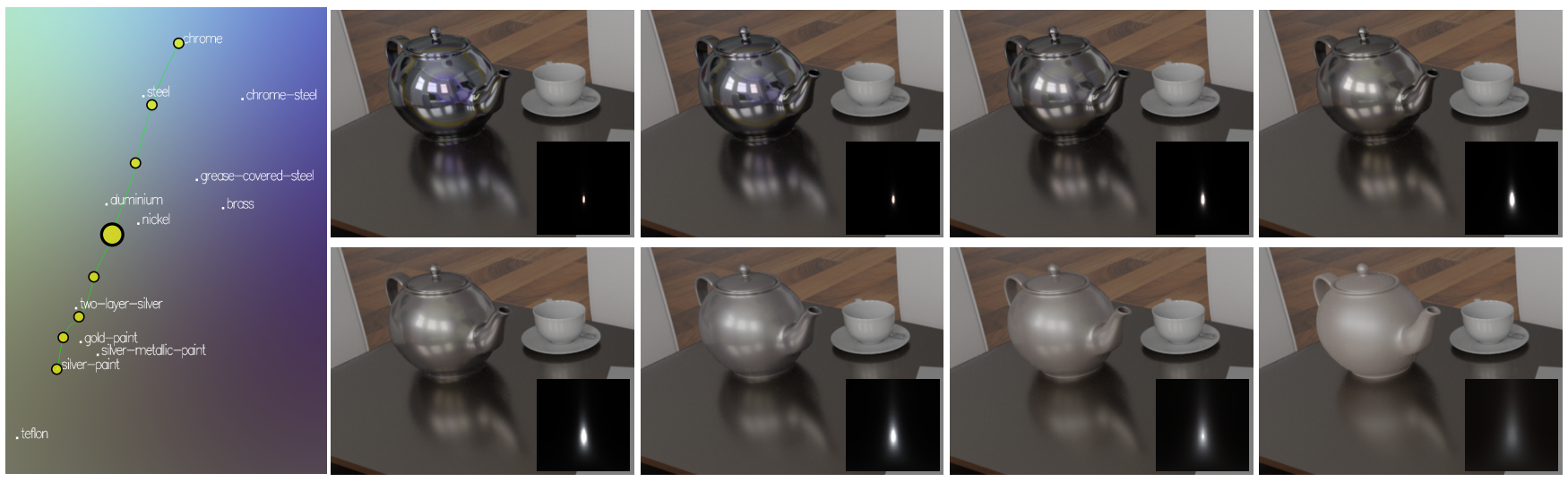

Figure 12: Snapshots captured during a live material editing session where the material on the teapot was continuously interpolated between MERL chrome and Silver-paint, in a $5 D$ latent space. Slices of the corresponding BRDFs are displayed as insets. Only the first (top left) and last (bottom right) images were pre-rendered with full global illumination using Mitsuba [Jak10]. Other images were computed in real-time using linear interpolation of pre-rendered images using our technique to compute blending weights. Note the consistent change in the reflection of the teapot on the table. The bigger dot corresponds to the material used for the error test in Figure 13. Note: the albedo displayed is for the $2 D$ slice and therefore may not correspond to the materials which white dots are obtained after projection in $2 D$.
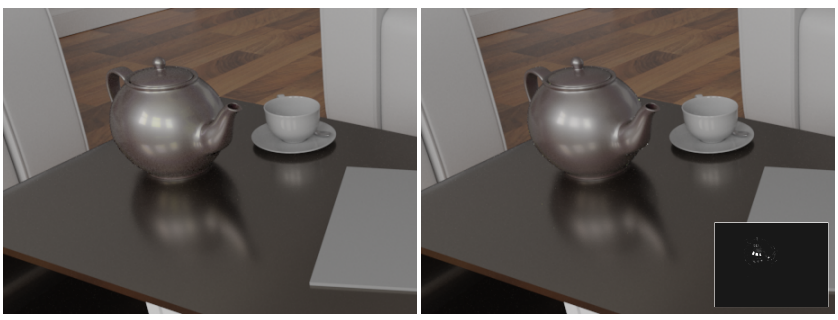

Figure 13: Comparison of our blended image (left), against a reference (right) computed with global illumination (using the corresponding interpolated reflectance data) for the midpoint along the path shown in Fig.12. The differences, due to the absence of $L_{2+}$ paths in our solution, are barely perceivable (well below the numerical error between the two images in the difference inset image).

near any chosen path. The optimization which produces the latent space will naturally cluster materials belonging to a coherent class (e.g. metals), with the benefit that interpolated materials will share characteristics of materials at nearby points. When the training data contains many different features (color, material class, shininess), it would be preferable to choose a sufficiently high dimensionality, allowing the optimizer to consistently cluster input data. We recommend $d=5$ for the entire MERL database.

Noisy measurements: In situations where the training data is noisy, the smoothing effect resulting from using low-dimensional representations can sometimes be an advantage. To benefit from this smoothing, with our representation, the $\mu$ in the covariance function of Equation 5 needs to be increased. However, our derivations for stability (ignoring $\mu$ ) then become increasingly conservative.

\section{Conclusion}

We have presented a method for learning and traversing a non-linear manifold of measured BRDFs. The input to our method is a set of reflectivity measurements made at locations in the 4D domain of
BRDFs. The locations are obtained by densely sampling the space composed of incident and exitant angles. First we obtain the mapping from the measurement space $(\mathrm{d}=4 \mathrm{M})$ to a much smaller latent space $(\mathrm{q}=2)$. For novel points in this latent space, obtained by interpolating the latent variables associated with the measured BRDFs, we use the mapping to calculate the corresponding high-dimensional point. The computed high-dimensional point corresponds to the virtual measurements associated with the interpolated latent variable. The key property of our method is that these virtual measurements express as linear combinations of the measured data. We combine this with existing techniques to enable real-time rendering and fast blending of precomputed images with global illumination, for objects with interpolated materials. An exciting application, for future work, will be to extend recent work on BRDF inference [GVPG15] using our representation to perform single-shot BRDF acquisition.

\section{Acknowledgements}

We thank the anonymous EuroGraphics reviewers for valuable suggestions towards improving this paper. Kartic Subr was funded by the Royal Society's University Research Fellowship. Cyril Soler was supported by ANR-16-CE33-0026 "CaLiTrOp".

\section{References}

[AAl16] Aittala M., Aila T., Lehtinen J.: Reflectance modeling by neural texture synthesis. ACM Trans. Graph. 35, 4 (July 2016), 65:165:13. 2

[AP07] Ashikhmin M., Premoze S.: Distribution-based BRDFs. Tech. rep., Dep. of Computer Science, University of Utah, March 2007. 2

[BOK11] Bilgili A., ÖZtürk A., Kurt M.: A general BRDF representation based on tensor decomposition. Comput. Graph. Forum 30, 8 (2011). 2

[BSH12] Bagher M. M., Soler C., Holzschuch N.: Accurate fitting of measured reflectances using a Shifted Gamma micro-facet distribution. Computer Graphics Forum 31, 4 (June 2012), 1509-1518. 2

[BSN16] Bagher M. M., Snyder J., Nowrouzezahrai D.: A nonparametric factor microfacet model for isotropic brdfs. ACM Transactions on Graphics 36, 6 (Aug. 2016). 2 
[BvdPPH11] Bonneel N., Van De Panne M., Paris S., Heidrich W.: Displacement interpolation using lagrangian mass transport. ACM Trans. Graph. 30, 6 (Dec. 2011), 158:1-158:12. 7

[DRS08] Dorsey J., RushmeIER H., Sillion F.: Digital Modeling of Material Appearance. Morgan Kaufmann Inc., San Francisco, CA, USA, 2008. 2

[GGG*16] Guarnera D., Guarnera G., Ghosh A., Denk C., Glencross M.: Brdf representation and acquisition. Comput. Graph. Forum 35, 2 (May 2016), 625-650. 2

[GVPG15] Georgoulis S., Vanweddingen V., Proesmans M., Gool L. V.: A gaussian process latent variable model for brdf inference. In $\operatorname{ICCV}(2015)$. 2, 8, 9

[Har97] Harville D. A.: Matrix Algebra From a Statistician's Perspec tive. 1997. 4

[HJ61] HoOKE R., JEEVES T.: "direct search" solution of numerical and statistical problems. Journal of the Association for Computing Machinery (ACM) 8, 2 (1961), 212-229. 4

[HLW15] Hao J., LIU Y., Weng D.: A BRDF Representing Method Based on Gaussian Process. Springer International Publishing, Cham, 2015, pp. 542-553. 2, 7

[HP17] Holzschuch N., PaCANowski R.: A Two-Scale Microfacet Reflectance Model Combining Reflection and Diffraction. ACM Transactions on Graphics 36, 4 (July 2017), 12. Article 66. 2

[HSL*17] Hui Z., SunkaVAlli K., LeE J.-Y., HadAP S., WANG J., SANKARANARAYANAN A. C.: Reflectance capture using univariate sampling of brdfs. In The IEEE International Conference on Computer Vision (ICCV) (Oct 2017). 2

[Jak10] JАКОВ W.: Mitsuba renderer, 2010. http://www.mitsubarenderer.org. 9

[KM99] KAUTZ J., MCCOOL M. D.: Interactive rendering with arbitrary BRDFs using separable approximations. In Rendering Techniques (1999). 2,3

[KSS02] KAUTZ J., SLOAN P.-P., SNYDER J.: Fast, arbitrary BRDF shading for low-frequency lighting using spherical harmonics. In Proceedings of the 13th Eurographics Workshop on Rendering (Aire-la-Ville, Switzerland, Switzerland, 2002), EGRW '02, Eurographics Association, pp. 291-296. 3

[Law05] LAWRENCE N.: Probabilistic non-linear principal component analysis with gaussian process latent variable models. J. Mach. Learn. Res. 6 (Dec. 2005), 1783-1816. 3

[LBAD*06] Lawrence J., Ben-Artzi A., DeCoro C., Matusik W. Pfister H., RAmAmoorthi R., Rusinkiewicz S.: Inverse shade trees for non-parametric material representation and editing. In $A C M$ SIGGRAPH 2006 Papers (New York, NY, USA, 2006), SIGGRAPH '06, ACM, pp. 735-745. 2

[LKG*03] Lensch H. P. A., Kautz J., Goesele M., Heidrich W., SEIDEL H.-P.: Image-based reconstruction of spatial appearance and geometric detail. ACM Trans. Graph. 22, 2 (Apr. 2003), 234-257. 2

[LKYU12] LÖW J., KRONANDER J., YNNERMAN A., UNGER J.: BRDF models for accurate and efficient rendering of glossy surfaces. ACM Transactions on Graphics (TOG) 31, 1 (January 2012), 9:1-9:14. 2

[LRR04] LAWRENCE J., RUSINKIEWICZ S., RAMAMOORTHI R.: Efficient BRDF importance sampling using a factored representation. ACM Trans. Graph. 23, 3 (Aug. 2004), 496-505. 2

[MPBM03] Matusik W., Pfister H., Brand M., McMillan L.: A data-driven reflectance model. In ACM SIGGRAPH 2003 Papers (New York, NY, USA, 2003), SIGGRAPH '03, ACM, pp. 759-769. 1, 2, 4, 6, 7

[MWL*99] Marschner S. R., WESTIN S. H., LAFORTUNE E. P. F., TORRANCE K. E., GREENBERG D. P.: Image-based BRDF measurement including human skin. In Proceedings of the 10th Eurographics Conference on Rendering (Aire-la-Ville, Switzerland, Switzerland, 1999), EGWR'99, Eurographics Association, pp. 131-144. 2
[NDM05] NGAN A., DURAND F., MATUSIK W.: Experimental analysis of BRDF models. In Proceedings of the Eurographics Symposium on Rendering (2005), Eurographics Association, pp. 117-226. 2

[NLW*16] NAm G., LeE J. H., Wu H., Gutierrez D., Kim M. H.: Simultaneous acquisition of microscale reflectance and normals. $A C M$ Trans. Graph. 35, 6 (Nov. 2016), 185:1-185:11. 2

[PFG00] Pellacini F., Ferwerda J. A., Greenberg D. P.: Toward a psychophysically-based light reflection model for image synthesis. In Proceedings of the 27th Annual Conference on Computer Graphics and Interactive Techniques (New York, NY, USA, 2000), SIGGRAPH '00, ACM Press/Addison-Wesley Publishing Co., pp. 55-64. 3

[PR12] PEREIRA T., RUSINKIEWICZ S.: Gamut mapping spatially varying reflectance with an improved BRDF similarity metric. Computer Graphics Forum (Proc. Eurographics Symposium on Rendering) 31, 4 (June 2012). 6

[PTVF07] Press W. H., Teukolsky S. A., Vetterling W. T., FlanNERY B. P.: Numerical Recipes 3rd Edition: The Art of Scientific Computing, 3 ed. Cambridge Univ. Press, New York, NY, USA, 2007. 4

[Ram09] RAmAmoorthi R.: Precomputation-based rendering. Found. Trends. Comput. Graph. Vis. 3, 4 (Apr. 2009), 281-369. 3

[RW06] Rasmussen C. E., Williams C.: Gaussian Processes for Machine Learning. 2006. 3, 4

[SBN15] Soler C., BAgher M., Nowrouzezahrai D.: Efficient and Accurate Spherical Kernel Integrals using Isotropic Decomposition. ACM Transactions on Graphics 34, 5 (Nov. 2015), 14. 2, 3, 7, 8

[SGM*16] Serrano A., Gutierrez D., MyszKowski K., Seidel H.-P., MASIA B.: An intuitive control space for material appearance. ACM Trans. Graph. 35, 6 (Nov. 2016), 186:1-186:12. 3, 8

[SKS02] SloAn P.-P., Kautz J., SNyder J.: Precomputed radiance transfer for real-time rendering in dynamic, low-frequency lighting environments. ACM Trans. Graph. 21, 3 (July 2002), 527-536. 3

[SM02] Steigleder M., McCool M. D.: Factorization of the Ashikhmin BRDF for real-time rendering. J. Graphics, GPU, \& Game Tools 7, 4 (2002), 61-67. 2

[SS95] Schröder P., SwEldens W.: Spherical wavelets: Efficiently representing functions on the sphere. In Proceedings of the 22Nd Annual Conference on Computer Graphics and Interactive Techniques (New York, NY, USA, 1995), SIGGRAPH '95, ACM, pp. 161-172. 2

[SZC*07] Sun X., ZHOU K., Chen Y., Lin S., Shi J., Guo B.: Interactive relighting with dynamic brdfs. ACM Trans. Graph. 26, 3 (July 2007)

[WAKB09] Wills J., Agarwal S., Kriegman D., Belongie S Toward a perceptual space for gloss. ACM Trans. Graph. 28, 4 (Sept. 2009), 103:1-103:15. 2, 3

[WAT92] Westin S. H., Arvo J. R., Torrance K. E.: Predicting reflectance functions from complex surfaces. In Proceedings of the 19th Annual Conference on Computer Graphics and Interactive Techniques (New York, NY, USA, 1992), SIGGRAPH '92, ACM, pp. 255-264. 2

[WdBKK15] WeINMANN M., DEN BRoK D., KRUMPEN S., KLEIN R. Appearance capture and modeling. In SIGGRAPH Asia 2015 Courses (2015), ACM. 2

[WM01] Westlund H. B., Meyer G. W.: Applying appearance standards to light reflection models. In Proceedings of the 28th Annual Conference on Computer Graphics and Interactive Techniques (New York, NY, USA, 2001), SIGGRAPH '01, ACM, pp. 501-51. 3

[WMLT07] Walter B., Marschner S. R., Li H., TorRance K. E.: Microfacet models for refraction through rough surfaces. In Rendering Techniques (2007), Eurographics Association, pp. 195-206. 2

[XCM*14] XU K., CaO Y.-P., Ma L.-Q., Dong Z., Wang R., Hu S.M.: A practical algorithm for rendering interreflections with all-frequency brdfs. ACM Trans. Graph. 33, 1 (Feb. 2014), 10:1-10:16. 3

[XNY*16] XU Z., Nielsen J. B., YU J., Jensen H. W., RAMAMoorTHI R.: Minimal brdf sampling for two-shot near-field reflectance acquisition. ACM Trans. Graph. 35, 6 (Nov. 2016), 188:1-188:12. 2 